\title{
Slowdown intracranial glioma progression by optical hyperthermia therapy: study on a CT-2A mouse astrocytoma model
}

\author{
Oscar Casanova-Carvajal Ana Lorena Urbano-Bojorge \\ Milagros Ramos José Javier Serrano-Olmedo and \\ R Martínez-Murillo
}

\begin{abstract}
Metallic nanorods are promising agents for a wide range of biomedical applications. We report an optical hyperthermia method capable of inducing slowdown tumor progression of an experimental in vivo CT-2A glioblastoma tumor. The tumor model used in this research is based on the transplantation of mouse astrocytoma CT-2A cells in the striatum of mice by intracranial stereotaxic surgery. Two weeks after cell implant, the resulting tumor is treated by irradiating intratumoral injected gold nanorods, biofunctionalized with CD133 antibody (B-GNRs), using a continuous wave laser. Nanoparticles convert the absorbed light into localized heat (reaching up to $44{ }^{\circ} \mathrm{C}$ ) due to the effect of surface plasmon resonance. A significant slowdown in CT-2A tumor progression is evident, by histology and magnetic resonance imaging, at one $(p=0.03)$ and two weeks $(p=0.008)$ after irradiation treatment. A notable deceleration in tumor size $(15 \%-75 \%)$ as compared to the control untreated groups, it is observed. Thus, laser irradiation of B-GNRs is found to be effective for the treatment of CT-2A tumor progression. Similarities between the pre-clinical CT-2A tumor model and the human astrocytoma disease, in terms of anatomy, metastatic behavior and histopathology, suggest that hyperthermic treatment by laser irradiation of B-GNRs administered into high-grade human astrocytoma might constitute a promising alternative treatment to limit the progression of this deadly disease.
\end{abstract}

Keywords: CT-2A cells, glioblastoma, gold nanorods, hyperthermia, syngeneic mouse

(Some figures may appear in colour only in the online journal)

\section{Introduction}

Cancer is the second leading cause of death in the world; in 2015 caused 8.8 million deaths. According to the World Health Organization [1] nearly one out of six deaths in the world is due to this disease. Primary brain tumors represent one of the most challenging forms of neoplasia to be treated. High-grade gliomas, including glioblastoma multiform
(GBM) are the most aggressive type of primary brain tumor and despite medical intervention the median survival is only 12-15 months and 5 year survival is less than 5\% GMB [2-4]. Infiltrating cancer cells beyond the boundaries of the tumor edge are responsible for tumor recurrence as well as radiation and chemotherapy resistance [5-7].

In recent years nanomedicine has been progressing rapidly and defining it as the use of materials with dimensions 
in the nanometric scale range for a specific diagnostic or therapeutic purpose. These advances in nanotechnology have allowed the development of gold nanostructures with optical properties that are useful in biomedical applications like drug delivery, hyperthermia therapy, and image contrast agents due to their biocompatibility and optical tenability [8-11]. Gold nanoparticles are highly appealing for cancer diagnostics and therapy because gold is almost an inert material (high resistance to corrosion), it has low toxicity and it is easy to anchor biocompatible ligands to its surface [12].

Biofunctionalization of gold nanoparticles techniques are well-known procedures for biomedical technologies both in vitro and in vivo [13, 14]. Gold nanoparticles conjugates with specific antibodies for breast cancer have been recently used for hyperthermia in order to increase the specificity in anticancer treatments [8, 14-16]. Experimental neuro-oncology demands the creation of appropriate animal models to assess the efficacy of innovate approaches for the treatment of human tumors. This is the case of CT-2A astrocytoma model, this cellular model has advantages compared to cellular lines such as U87, where the high proliferation of CT2A was evidenced, being these more invasive [14-18]. CT-2A astrocytoma model was used for therapeutic screening in preclinical trials in this study. Imaging technologies in the CT-2A model measuring tumor growth would provide insights on gold nanorods (GNRs), biofunctionalized with CD133 antibody (B-GNRs), efficacy (see section 4.5). The CT-2A cell line overexpresses the membrane receptor CD133. Recently, CD133 antibodies have been employed in nanomedicine, oriented towards hepatocellular carcinoma [19]. Experimental neuro-oncology demands the creation of appropriate animal models to assess the efficacy of innovate approaches for the treatment of primary human brain tumors [20, 21]. Due to similarity of the CT-2A tumor model to human astrocytoma disease in terms of anatomy, metastatic behavior and histopathology [17, 18], this mouse model becomes an excellent candidate to assay new anticancer procedures.

Based on using B-GNRs, together with laser irradiation ( $808 \mathrm{~nm}$ wavelength continuous wave laser source), we present here evidence that our hyperthermia procedure provides basis for a new anti GBM therapy. This paper reports for the first time beneficious effects of this technology limiting intracranial CT-2A mouse astrocytoma tumor progression. The B-GNRs used in the present study showed high cytocompatibility, as previously reported in vitro $[19,22]$. The tumor biology of the CT-2A immunocompetent glioblastoma murine model, derived from a malignant astrocytoma originally formed after the intracerebral implantation of 20methylcholanthrene pellets into C57/BL6 mice [23], shows close similarities with the human glioblastoma disease thus becoming an excellent candidate to assay new anticancer procedures.

In this study, C57BL/6 mice were stereotaxically inoculated with CT-2A glioblastoma cells, administered directly in the striatum. After two weeks, the resulting tumors were treated with the optical hyperthermia technique described below and the tumor volumes analyzed by in vivo MRI.
The data show arresting in tumor growth in treated mice as compared with their respective controls.

Glioblastoma is highly infiltrative, thus resection is problematic and recurrence frequent. The photothermal treatment would complement the resection surgery of the solid tumor mass by treating infiltrated tumor cells within tumor environment. Essays combining surgical resection of tumors and optical hyperthermia treatment, are also in course.

\section{Results and discussion}

The experiments have been performed in a characterized CT$2 \mathrm{~A}$ orthotopic model of malignant mouse astrocytoma[17, 18] as result of intracerebral injection of the cell line CT-2A [23] into the caudateputamen (CP). The resulting CT-2A malignant tumor exhibited characteristics of human high-grade astrocytoma, as shown by anatomy, histopathology, and noninvasive imaging procedures (MRI) (figures 3 and 4 and 6 , and 8). Tumor-host Interactions are a far-reaching relationship and extend well beyond the local tissue microenvironment, thus mouse models should be orthotopic to better reproduce tumor biology and should also consider the contribution of the immune system. For these reasons, the chosen CT-2A tumor model in this study was orthotopic and accomplished in immunocompetent syngeneic mice.

\subsection{Tumor growth}

The characteristics of CT-2A malignant cells, both in vitro and in vivo, were described elsewhere [17, 18]. Cells grew in vitro attached to the substrate as a monolayer, a characteristic essential for their proliferation which stops once they became confluent. Adherent cells exhibited an elongated shape. In vivo, the CT-2A tumor consisted of a homogeneous hypercellular mass with well-defined borders (figures 3 and 4, and $6(\mathrm{~A})$ ). One week after intracranial transplantation, a soft cell mass was observed into the injected area of brain parenchyma (figures 3 and 6). The angiogenic, hemorrhagic and infiltrative pattern of the CT-2A tumor was confirmed three to four weeks following cell implant (figures 4 and 6). At that time, metastatic cells were located at more distant sites, resulting in satellite lesions. Characteristic features of a highgrade glioma, including high mitotic activity, cellular density, microvascular proliferation, spontaneous hemorrhagic regions and necrosis (figures 3 and 4, and 6), were confirmed as previously reported by our laboratory [17]. The ultrastructure of CT-2A cells revealed characteristics of malignant cells. Neoplastic cells were pleomorphic with either large fusiform or small round shape, with prominent nuclei exhibiting areas of heterochromatin and prominent nucleoli (figure 5). The cytoplasm contained abundant relatively well developed Golgi apparatus, well conserved mitochondria and polyribosomes, indicating a high metabolic activity. Abundant interstitial space between tumor cells was also a characteristic of the CT-2A tumor, suggesting interstitial fluid drainage alterations in the tumor mass. 


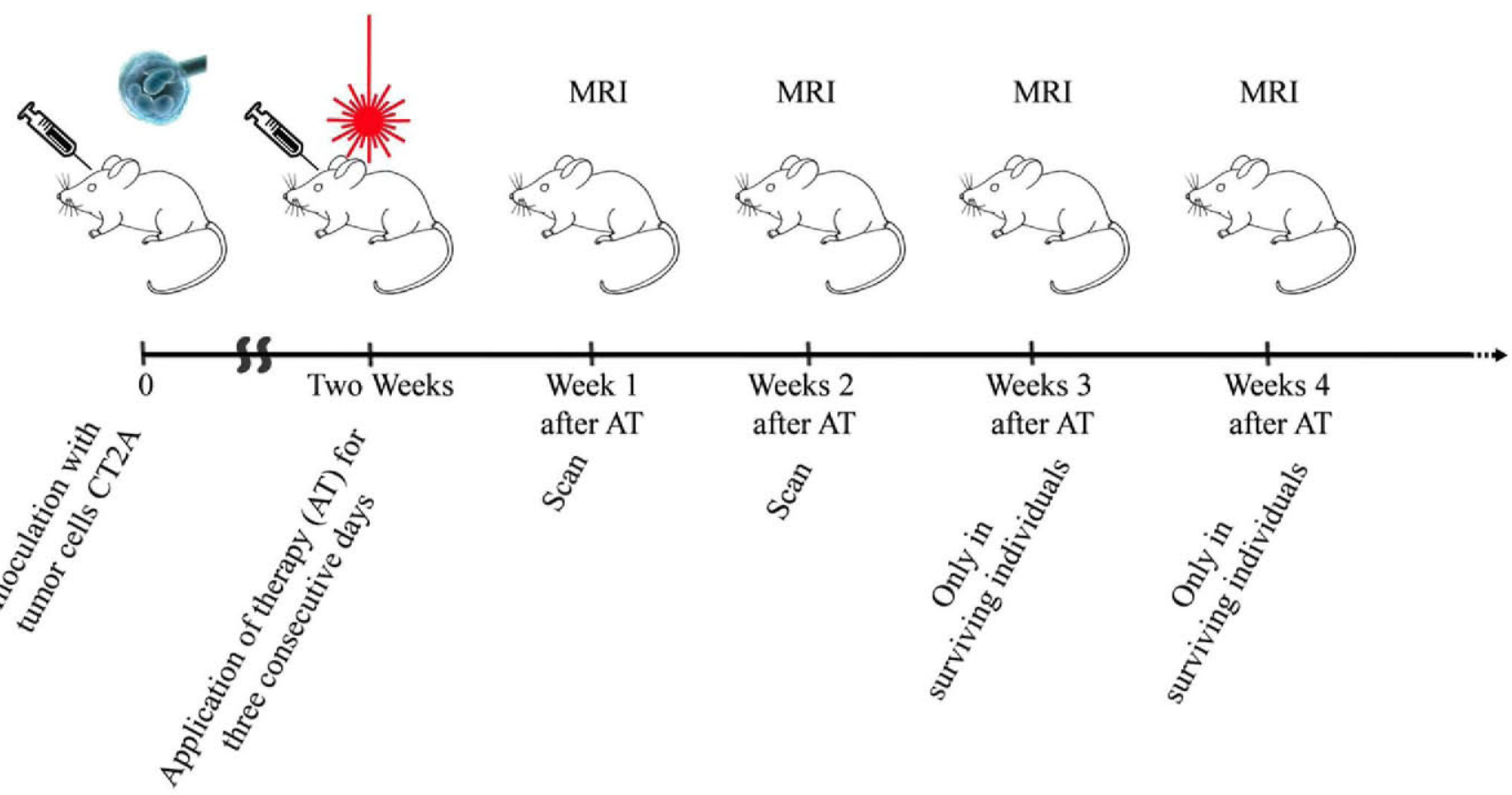

Figure 1. Summary graph of the methodology.

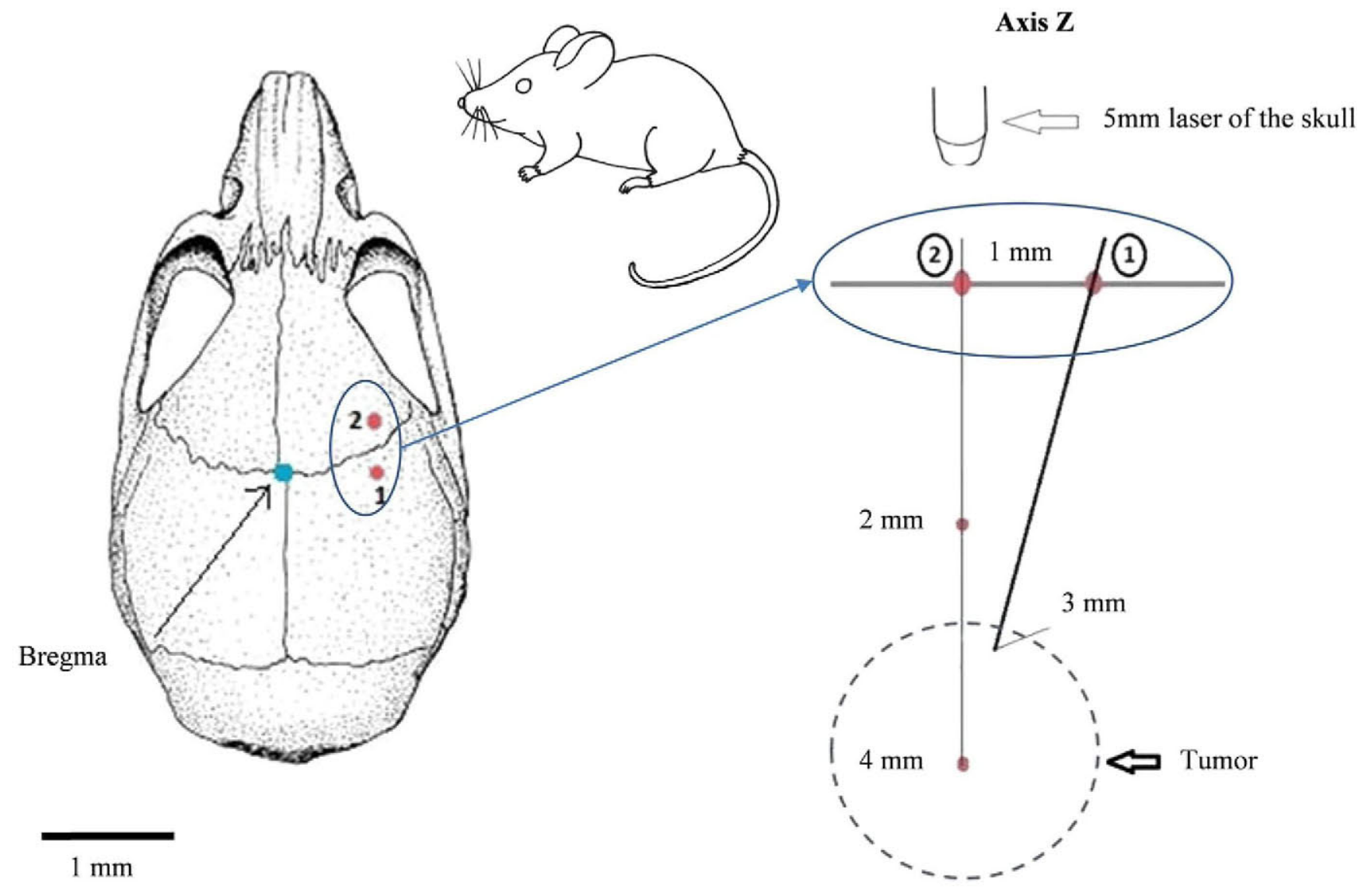

Figure 2. Methodology of hyperthermic temperature measurements.

\subsection{MRI analysis}

MRI is the most commonly used diagnostic tool for glioblastoma multiforme. In this study, T1-weighted post-contrast (gadolinium) MRI procedure was carried out as a tool to obtain precise images of the tumor vascularity, with a resolution limit of $0.8-1 \mathrm{~mm}^{3}$. This information is needed to delineate tumor boundaries for assessment of intracranial tumor location and growth pattern evaluation (figures 3(A), 

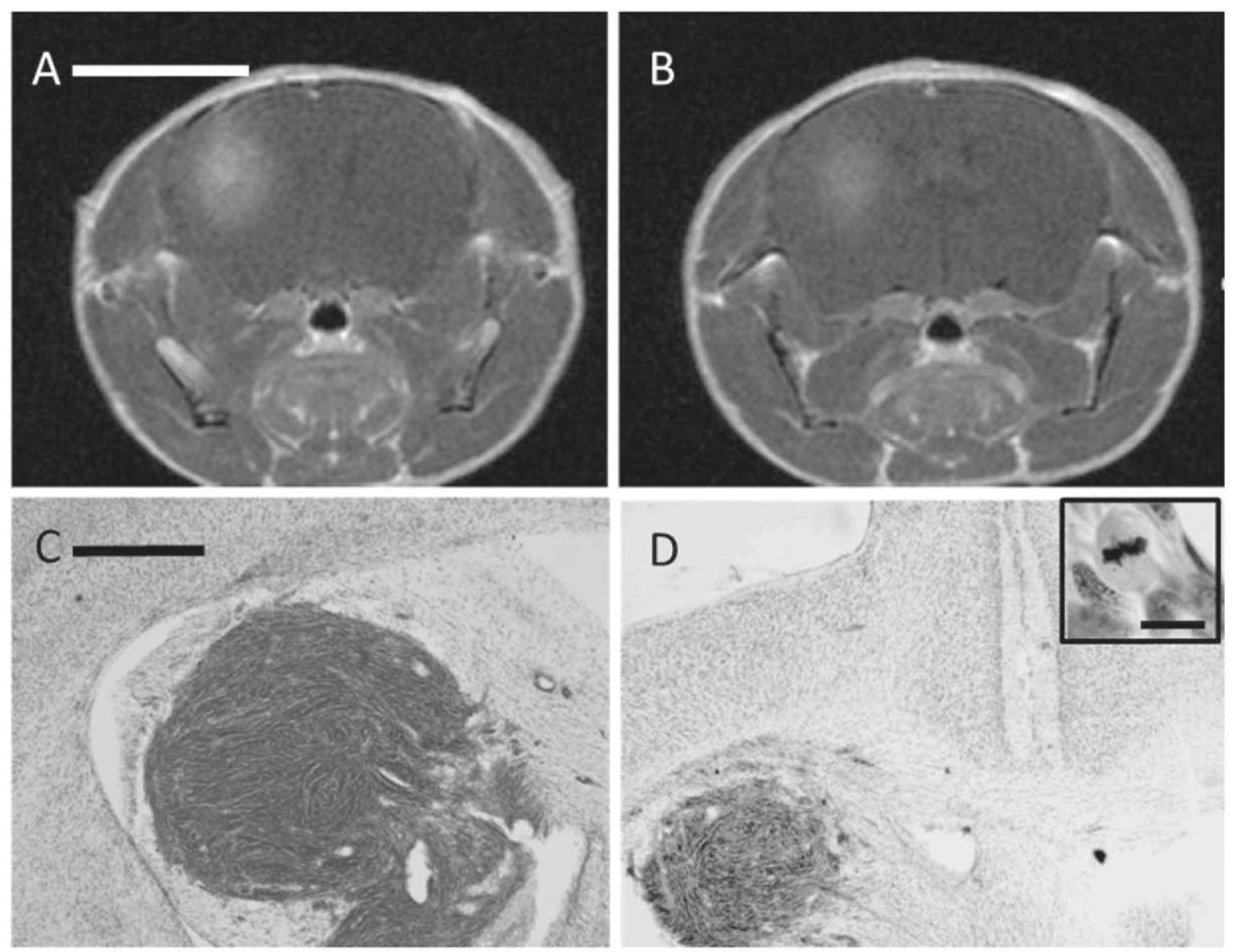

Figure 3. Cell proliferation as seen by MRI and H\&E staining. Panels (A), (B), represent T1-weighted (Gadolinium postcontrast) images corresponding to the same experimental CT-2A tumor depicted in (C), (D), respectively, showing the tumor progression at different rostrocaudal levels, two weeks following cell implant. Notice that in (A) and (B) the hyperintense signal is restricted to the CP in the right hemisphere. Inset in (D) shows a mitotic image nearby a neoformed blood vessel. Scale bars: $A, B=5 \mathrm{~mm} ; \mathrm{C}, \mathrm{D}=1 \mathrm{~mm}$, inset in $\mathrm{D}=15 \mu \mathrm{m}$.
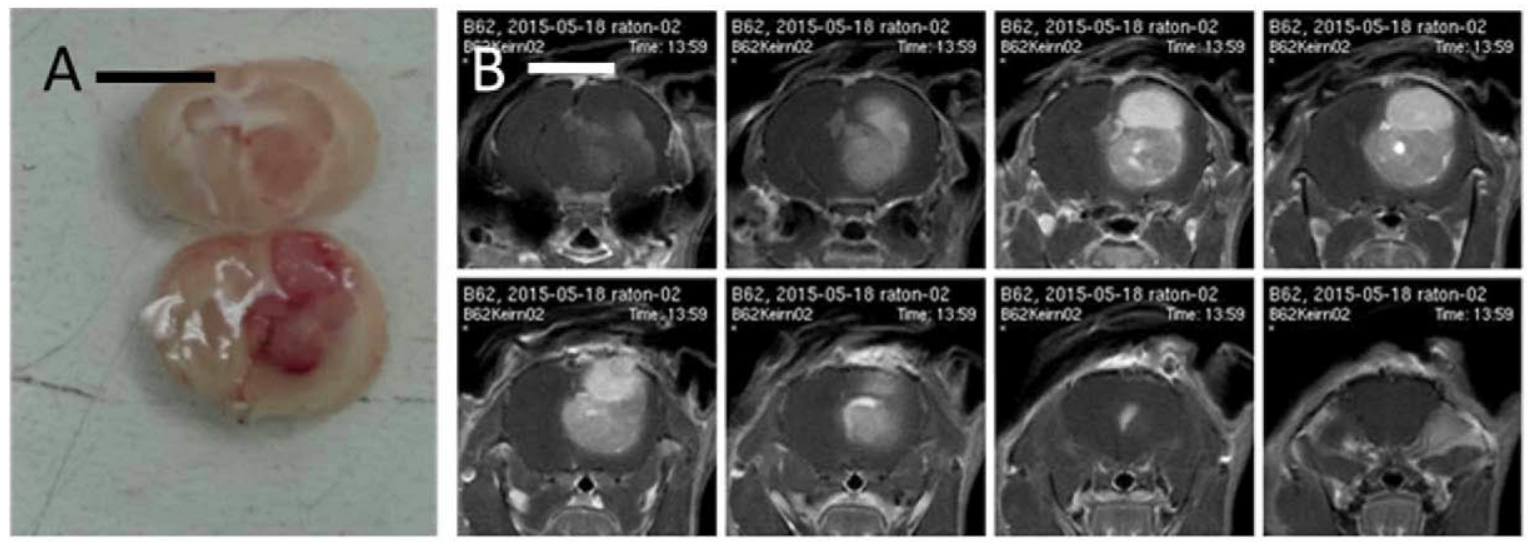

Figure 4. Characterization of CT-2A induced brain tumors. (A) Coronal $1 \mathrm{~mm}$ thick coronal sections through the same mouse brain with a hemorrhagic CT-2A tumor mass lying in the right cerebral hemisphere, $28 \mathrm{~d}$ following CT-2A cell injection. The photograph was taken after in vivo MRI (B) and removal of the brain at the time of necropsy for histology. (B) Panels represent T1-weighted (Gadolinium postcontrast) images at different rostro-caudal levels corresponding to the same experimental CT-2A tumor depicted in (A), showing the tumor progression four weeks following cell implant. Notice in (B) hyperintense signal in the rim of the tumor indicating that the tumor border is wellvascularized and also hypo-attenuation in central regions of the tumor suggesting necrosis. Scale bars: $\mathrm{A}, \mathrm{B}=4 \mathrm{~mm}$. 

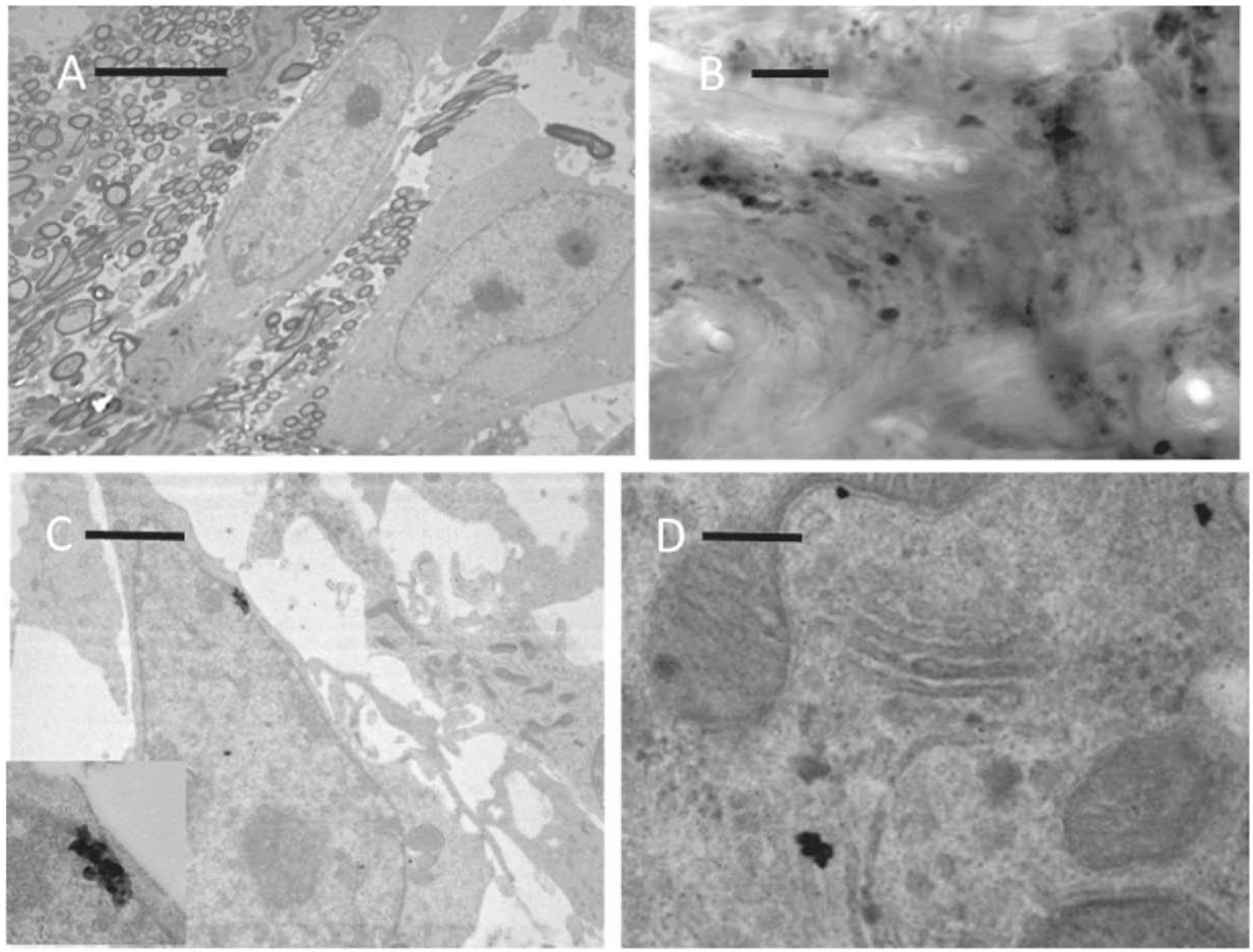

Figure 5. (A) Electron microscopic image of osmium-stained CT-2A tumor cells illustrating the ultrastructural appearance of tumor cells in the transition zone with the striatum. Tumor cells show cytological pleomorphism with prominent nucleoli, slender processes and characteristic heterochromatin. Notice adjacent myelinic fibers of the striatum in the surrounding neuropil and apparent edema, suggesting inflammation of the tissue. (B) Light micrograph showing intratumoral B-GNRs deposition attached to tumor cells, mainly nearby capillaries, as revealed by immunohistochemical staining. (C) and (D), Illustrate deposition of the intratumoral injected B-GNRs. B-GNRs are detected as electron-dense complexes that are attached to the nuclear membrane (C) and also dispersed in the cytoplasm (D). Inset in (C), is a higher power magnification of the electron-dense product shown in (C). Scale bars: $\mathrm{A}=15 \mu \mathrm{m} ; \mathrm{B}=10 \mu \mathrm{m} ; \mathrm{C}=5 \mu \mathrm{m} ; \mathrm{D}=0,25 \mu \mathrm{m}$.

(B), and 4(B) and 7, and 8). Evaluation by MRI of tumor limits correlated accurately with the histopathological location of CT-2A cells (figures 3 and 4). Characteristically, T1weighted images marked the increase of angiogenesis in the tumor limits which helps to delineate precisely the tumor margins (figure 4(B)). T1-weighted imaging to verify tumor growth and location was routinely processed every week (figures 7 and 8). MRI images revealed that the tumor mass arose initially in the $\mathrm{CP}$ (figures 3(A), (B) and 7(A)), spreading over time rostro-caudally, eventually involving surrounding areas of the $\mathrm{CP}$, including cerebral cortex and hippocampus (figures 4(B) and 8(B), (D)). Tumor mass was barely detectable one week post-transplant, reaching an unbearable status at 4 weeks (figures 4(B) and 7(B)). At this time post implantation, the tumor growth resulted in marked deformation of surrounding brain structures in mice showing signs of intracranial pressure and neurological complications. Hypointense areas in the center on T1-weighted enhanced images, suggesting central necrosis, were frequently seen (figures 4(B) and 8(B), (D)), that were confirmed by histopathology in all cases. Experimental animals were sacrificed when signs of motor dysfunction arise or exhibited symptoms consistent with failure to thrive, as per animal care protocols that advised its sacrifice.

\subsection{Immunohistochemistry}

Light microscopy immunocytochemistry for CD133 and Ki67 revealed numerous positive CT-2A cells in the tumor mass (figures 6(A)-(C)). Ki67 shows intense nuclear staining (figures 6(A), (B)) whereas CD133 was exclusively cytoplasmatic, with the reaction product accumulated in puncta in the cytoplasm of tumor cells (figure 6(C)). Ki67 immunoreactive CT-2A in the tumor mass characteristically accumulated in the tumor edge and close to blood vessels (figures 6(A), (B)). Double immunofluorescence for BrdU and Nestin revealed that whereas BrdU-positive cells were abundant in the tumor mass, Nestin-positive cells also exhibiting BrdU staining were exclusively located in tumor endothelial cells (figure 6(D)). The latter correlated with previous studies that Nestin is a marker for proliferative endothelium in gliomas [24]. We can therefore conclude that the preclinical astrocytoma CT-2A mouse model in this study 

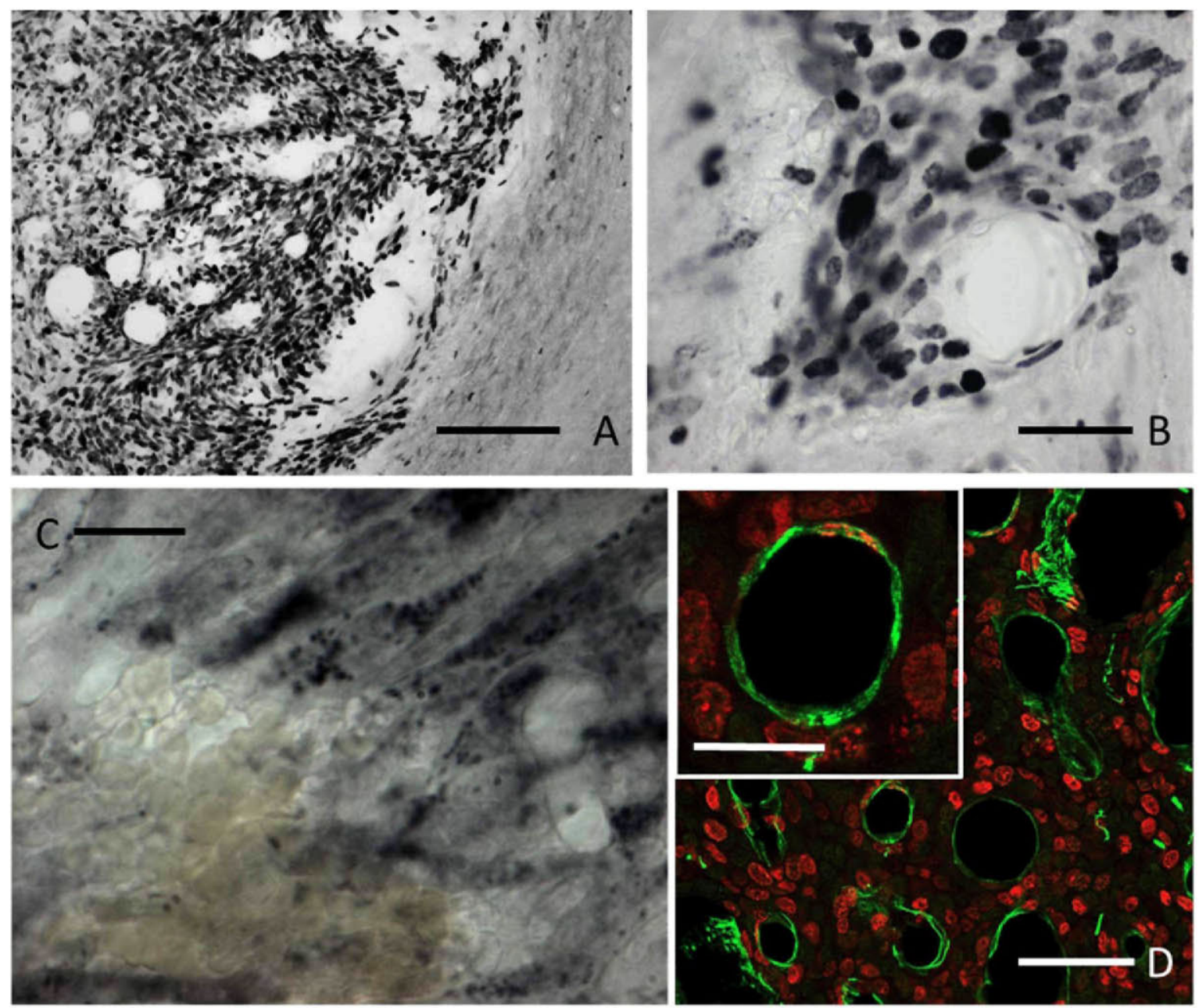

Figure 6. Immunocytochemical characterization of induced brain tumors in C57BL/6 mice, $21 \mathrm{~d}$ following CT-2A cell line implantation. Light micrographs (A)-(C), illustrate Ki67 (A), (B) and CD133 (C) positive cells in the tumor mass. Notice in (A) that numerous intratumoral blood vessels (asterisks) are seen in the tumor mass. Also in (B) that ki67-positive cells exhibit immunoreaction product in the cell nucleus of cells that characteristically arranged around a capillary. In addition, see in (C) puncta of CD133-immunoreaction product restricted to tumor cells. The yellow color at the bottom of the left hand side in (C) represents a hemorrhagic region. (D) Illustrates tumor-associated neoangiogenesis by double immunofluorescence staining for BrdU (cell nucleus, red) and Nestin (prolifetarive endothelium, green). As previously reported for human gliomas, Nestin-immunoreactivity was found as a marker in this study for proliferative endothelium in the CT$2 \mathrm{~A}$ model. Inset in (D), shows details of an anomalous proliferating capillary. Scaler bars: $\mathrm{A}=500 \mu \mathrm{m} ; \mathrm{B}=200 \mu \mathrm{m} ; \mathrm{C}=50 \mu \mathrm{m}$; $\mathrm{D}=100 \mu \mathrm{m} ;$ Inset in $\mathrm{D}=50 \mu \mathrm{m}$.

mimics the human high-grade astrocytoma disease in terms of anatomy, metastatic behavior and histopathology.

\subsection{Efficacy of biofunctionalization}

To test the efficacy of B-GNRs as a tool to bound tumor cells, we examined by immunohistochemistry the cellular location of B-GNRs in tissue sections (see section 4.5). For this, tissue sections were processed to detect endogenous CD133 using the sample resuspension of B-GNRs compound as primary antibody. Immunohistochemistry was carried out as shown for CD133 immunohistochemistry in section 4.4. (Immunohistochemistry). For this, resuspended B-GNRs compound, diluted $1: 100$ in PBS containing $0.2 \%$ Triton X-100 was used as primary antibody. Tumor sections, $40 \mu \mathrm{m}$ thick, were incubated overnight at $4{ }^{\circ} \mathrm{C}$ with continuous stirring. Then, tissue sections were thoroughly washed and processed as for
CD133 immunohistochemistry in section 4.4. By light microscopy, we found the reaction-end product labeling exclusively proliferating CT-2A cells, following the same cellular distribution as that observed for CD133 immunohistochemistry (figure 5(B)). The latter suggesting that GNRs consistently bear anti-CD133 antibodies.

\subsection{Electron microscopy}

Under the electron microscope, the subcellular location of intratumoral injected B-GNRs compound (GNRs-protein G-CD133 complex; see section 4.5) was carried out. Occasional rods of B-GNRs electron-dense product bound to the nuclear membrane, whereas the majority of the B-GNRs deposits were associated with intracellular organelles in the cytoplasm (figures 5(C), (D), and inset in (C)). 


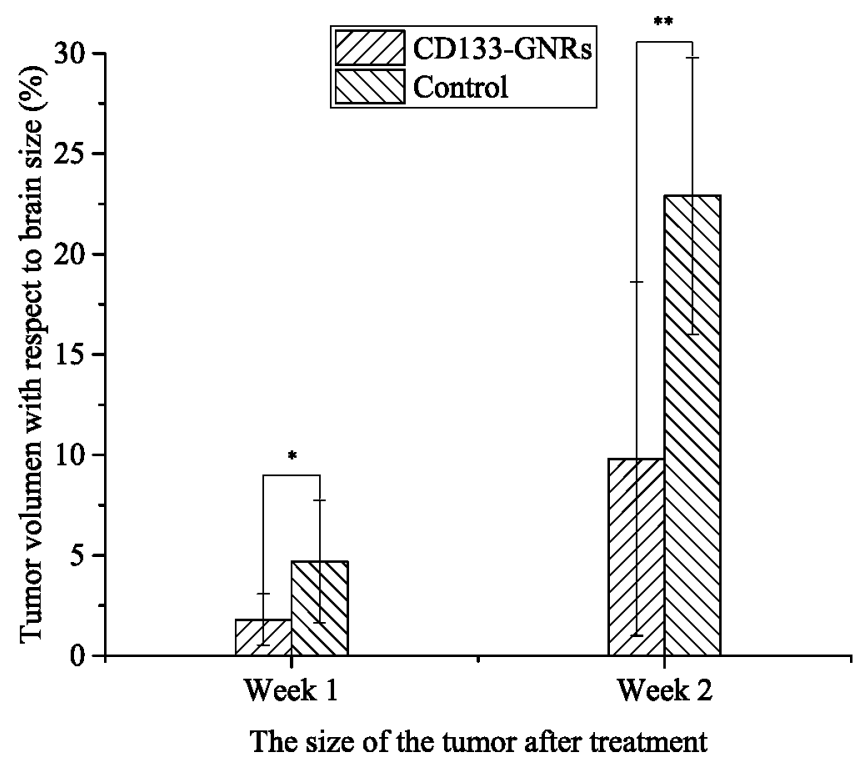

Figure 7. Tumor volume, expressed as a percentage of total brain volume, in control mice and B-GNRs + Laser (CD133-GNRs), at Week 1 and Week 2 (time periods after laser irradiation). The data represent the mean $\pm \mathrm{SD}$ of $n=3$ independent experiments. Student-t test revealed a significant difference $(p=0.03)$ between Control and CD133-GNRs at Week 1. The CD133-GNRs group reached higher significance benefit ${ }^{* *}$ at Week $2(p=0.008)$.

\subsection{Temperature measurements}

in order to corroborate the generation of an adequate increase in temperature to eliminate cells by hyperthermia, changes in temperature were determined in situ, after the injection of the B-GNRs as described in section 4.8. The results shown a temperature increase up to $40.0^{\circ} \mathrm{C} \pm$ $0.5^{\circ} \mathrm{C}$ in the control group (figure 9 ). In contrast, a increase of the temperature up to $43.0^{\circ} \mathrm{C} \pm 1.0^{\circ} \mathrm{C}$ was evidenced in the group injected with B-GNRs and irradiated with laser, using the same methodology in all the variables the dosemetric was $25 \mathrm{CEM} 43$. The measurements were made for a time of $25 \mathrm{~min}$. These values were in agreement with results inprevious reports $[25,26]$.

\subsection{Photothermal therapy}

Tumor growth was analyzed at two time points: one week after laser irradiation, that is to say, threeweeks after cell implant (Week 1), and two weeks after laser irradiation, that means, four weeks after cell implant (Week 2). In other words, $168 \mathrm{~h}$ and $336 \mathrm{~h}$, after laser irradiation of endogenously administered B-GNRs (CD133-GNR, 18 animals) and control PBS + Laser (Control, 9 animals). Figure 7 shows differences in tumor volume ratios between the CD133-GNRs and control groups which provide information about the tumor evolution as a percentage of the tumor volume with respect to the total brain volume. The tumor volume was measured by MRI at different post-treatment times: 'Week 1' and 'Week 2', following control (PBS + Laser) and
B-GNRs + Laser (CD133-GNRs) post-treatments (figure 8). At Week 1, CD133-GNRs group showed a significant reduction in tumor volume with regard to control $(p=0.03)$ : $1.8 \%$ versus $4.7 \%$, with respect to the brain size. It is noteworthy that differences in tumor progression between these group became highly significant $(p=0.008)$ at Week $2: 8.1 \%$ versus 22.9 with respect to the brain size. These data support the idea that intratumoral CD133-GNRs +Laser treatment exerts a potent tumoricidal effect in the CT-2A mouse astrocytoma model. Interestingly, two mice survived for more than 3 weeks after treatment, in separate experiments.

In this study, B-GNRs + laser treated mice showed a clear behavior of tumor reduction. Tumors were analyzed in consecutive weeks 1 (three weeks after CT-2A implant) and 2 (four weeks after CT-2A implant), after the initiation of hyperthermia treatment (figure 8). Panels in figure 8 correspond to MRI of the same brain sections of three representative mice: control PBS+laser irradiation (figures 8(A), (B)), B-GNRs without laser irradiation (figures 8(C), (D)) and B-GNRs+laser irradiation (figures $8(\mathrm{E}),(\mathrm{F})$ ), acquired in weeks 1 (figures 8(A), (C), (E); three weeks after CT-2A implant), and 2 (figures 8(B), (D), (F); four weeks after CT$2 \mathrm{~A}$ implant). The initial tumor mass in the striatum was faint one week after B-GNRs+laser irradiation (figure $8(\mathrm{E})$ ) comparing to controls (figures 8(A), (C)). In the B-GNRs + laser group (figures $8(\mathrm{E}),(\mathrm{F})$ ) the tumor mass showed a progressive decline, from $0.68 \%$ in 'Week 1 ' $\left(3 \mathrm{~mm}^{3}\right)$, to $0.37 \%$ in 'Week 2' $\left(2 \mathrm{~mm}^{3}\right)$ and $0.22 \%$ in 'Week 3' $\left(0.99 \mathrm{~mm}^{3}\right)$, comparing the total brain size, reaching a minimum value of $0.17 \%$, four weeks after treatment.

\section{Conclusions}

This study shows the efficacy of B-GNRs + Laser treatment promoting the tumoricidal effect of the optical hyperthermia on an in vivo CT-2A astrocytoma model. This treatment constitutes a promising alternative choice to treat intracranial neoplasms in humans. Reduction of intracranial tumor volume following this procedure can be considered as an outstanding achievement in the treatment of this deadly cancer that opens new avenues in the comprehension and treatment of primary brain cancer, supporting future research in this field.

\section{Materials and methods}

\subsection{Cell culture}

CT-2A cell line was provided by Prof T N Seyfried (Boston, MA, USA). Dispersed cells proliferated in RPMI 1640 cell culture medium (Gibco, Invitrogen Co., Carlsbad, CA, USA) supplemented with $10 \%$ heat-inactivated fetal bovine serum in a $\mathrm{CO}_{2}$ incubator with humidified atmosphere containing $5 \% \mathrm{CO}_{2}$ at $37^{\circ} \mathrm{C}$. CT-2A cells usually propagate as a 

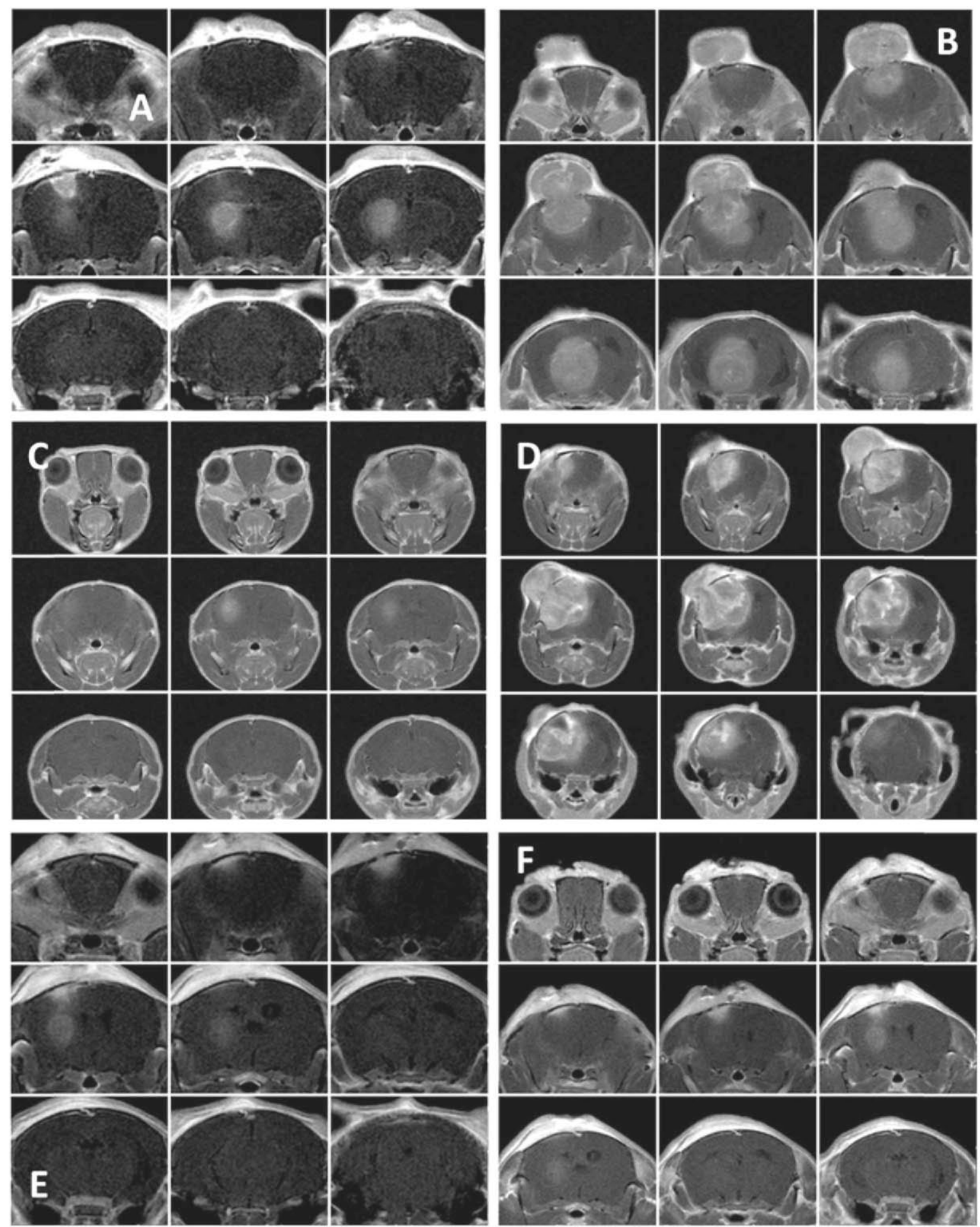

Figure 8. Shows MRI images of mice randomly chosen from groups: control (PBS + Laser) (A), (B), B-GNRs without laser (C), (D) and B-GNRs +Laser, for 'Week 1' (A), (C), (E) three weeks after CT-2A implant) and 'Week 2' (B), (D), (F) four weeks after CT-2A implant) post-treatments, respectively. These images show an apparent deceleration in tumor progression in the CD133-GNRs (B-GNRs) + Laser group.

monolayer attached to the substrate. The attachment of CT2A cells is essential for their proliferation. Upon reaching confluency, the cells were trypsinized and collected. The pellet was resuspended in RPMI 1640 medium and the cell suspension was adjusted to obtain an appropriate concentration of cells for injection $\left(8 \times 10^{4}\right.$ cells in $\left.4 \mu \mathrm{l}\right)$ [27].

\subsection{CT-2A tumor model}

A total of three experiments were conducted using adult C57BL $/ 6$ mice $(35.26 \mathrm{~g} \pm 1.01$ body weight). The experiments consisted of two groups: the treated group (CD133GNR, 18 animals) and the control group ( 9 animals). The control group was established by 3 animals injected with PBS 


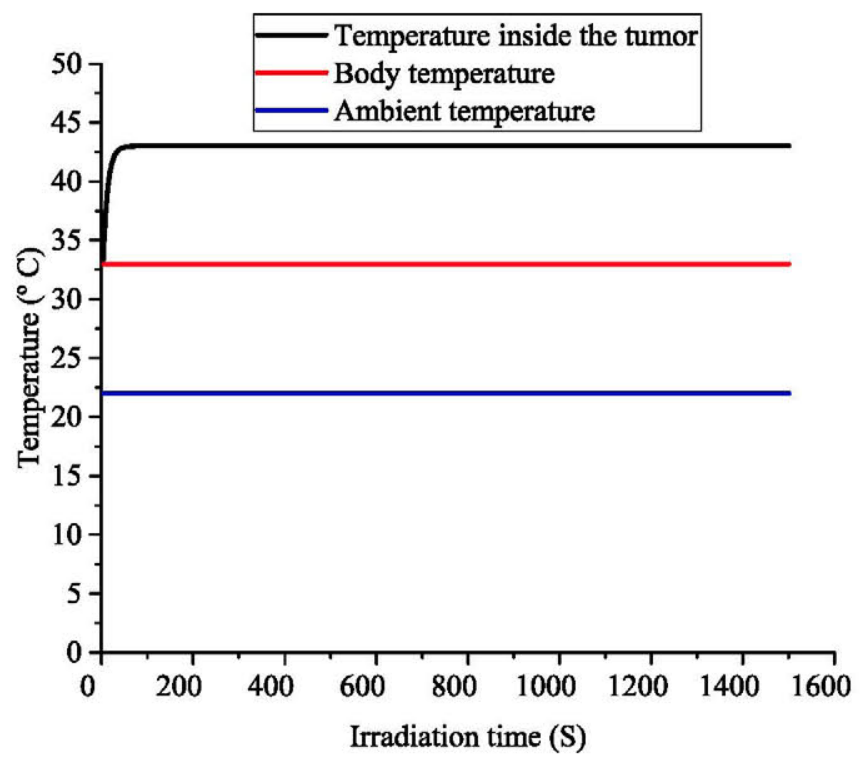

Figure 9. Hyperthermic temperature measurements.

and treated with laser, and 6 animals only injected with B-GNRs without laser (see figure 8 ). No statistically significant differences were detected between the control groups. It is worth noting that, it was merged into a single group. The animals were housed with free access to food and water in an animal room with a controlled temperature and a natural light cycle. Daily routines were performed between $7 \mathrm{a} . \mathrm{m}$. and $4 \mathrm{p}$. $\mathrm{m}$. by authorized personnel. CT-2A cells were implanted into the right $\mathrm{CP}$ by stereotaxic assistance (David Kopf Instruments, Tujunga, CA, USA). While under deep anesthesia, twenty-seven animals were operated following a previously reported procedure [17]. The tumor cells were injected in a volume of $0.15 \mu \mathrm{l}$ for a time frame of $15 \mathrm{~min}$, this amount was equivalent to approximately 3.000 cells. Two weeks after CT$2 \mathrm{~A}$ cell implant, cell proliferation was confirmed in vivo by T1-weighted gadolinium MRI, and postmortem by H\&E staining (figure 3). Surgical procedures were carried out by using a thermal blanket to keep under control the corporal temperature of the experimental animals to prevent the temperature from failing due to anesthesia. All procedures were performed under Spain and Spanish National Research Council (CSIC) Regulations for animal care (laws 53/2013 and ECC/566/2015).

\subsection{Histopathology}

Histopathology was performed on coronal tissue sections through the same rostro-caudal levels from fixed brains that were previously inspected by MRI as exhibiting tumor growth (figure 3). Deeply anesthetized mice were perfused transcardially through a blunt cannula with $20 \mathrm{ml}$ phosphatebuffered saline from Gibco (PBS) followed by $40 \mathrm{ml}$ of $4 \%$ paraformaldehyde (PF) in $0.1 \mathrm{M}$ phosphate buffer $(\mathrm{PB}), \mathrm{pH} 7$, 4. Brains were then dissected-out and immersed for $4 \mathrm{~h}$ in the same fixative, at room temperature. For $\mathrm{H}$ and $\mathrm{E}$ stain, tissue blocks were paraffin embedded and then cut coronally in
$5 \mu$ m thick sections (figures 3(B), (C)) with a microtome Leica Jung RM 2045.

\subsection{Immunohistochemistry}

For light microscopy immunohistochemistry, fixed brains were cryoprotected with $30 \%$ sucrose for $1 \mathrm{~d}$. After the brains sink, histological sections $40 \mu \mathrm{m}$ thick were then obtained in the coronal plane with the aid of cryostat (Leica CM1950). Endogenous CD133 protein expression in the CT-2A tumor was evinced by using polyclonal antibodies (St John's laboratories, STJ201168). To evaluate cell proliferation, Ki67 immunostaining was also carried out [28] by using polyclonal antibodies (ab15580 abecam). Immunohistochemistry was performed in free floating sections following the avidin-biotin-peroxidase complex (ABC) method [29]. After endogenous peroxidase blockade by preincubating tissue sections in $1 \% \mathrm{H}_{2} \mathrm{O}_{2}$ in PBS for one hour, the sections were incubated with $3 \%$ normal serum diluted in PBS containing for $1 \mathrm{~h}$ at room temperature (RT, and then incubated overnight at $4{ }^{\circ} \mathrm{C}$ in the primary antibodies diluted in PBS containing $0.2 \%$ Triton X-100. Antibodies dilutions were: (i) CD133, 1:500 and (ii) Ki67. Tissue sections were then washed in PBS and later processed for the (ABC) method [29] (Vector Laboratories, Burlingame, CA, USA) Peroxidase activity was developed by nickel enhanced 3,3'-diaminobenzidine tetrahydrochloride (DAB) procedure as described elsewhere [30]. Stained sections were later washed, mounted and dehydrated for light microscopy visualization with the help of a Zeiss Axiophot II microscope (Zeiss Iberica, Madrid, Spain) and images captured with a digital camera (DMC le, Polaroid, Cambridge, MA, USA). The method of BrdU labeling was also used for in vivo tumor cellular proliferation studies, following a procedure previously published by our laboratory [31]. As nestin was found as a marker for proliferative endothelium in gliomas [24], we combined nestin immunohistochemistry with $\mathrm{BdrU}$ immunostainig to test co-localizations of both antigens in the tumor model by immunofluorescence staining. Immunofluorescence staining was performed by incubating tissue sections in the specific antibodies for BrdU (rat, 1:2000, abcam) and nestin (rabbit, 1:000, Sigma-Aldrich, SAB4200347). After the sections were washed with PBS, primary antibodies were visualized using the corresponding Alexaconjugated secondary antibodies: Alexa Fluor ${ }^{(3)} 488$ donkey anti-rabbit, and Alexa Fluor ${ }^{(3)} 568$ goat anti-rat (1:1000, Invitrogen, Molecular Probes) for $2 \mathrm{~h}$ at RT and mounted on glass slides with anti-photobleaching mounting media (DABCO/ Mowiol). Sections were counterstained with 4,6-diamidino-2phenylindole (DAPI, $300 \mathrm{nM}$ in PBS, Invitrogen) and examined on a LEICA TCS SP5 scanner confocal microscope (Leica Microsystems GmbH, Wetzlar, Germany). Antibody specificity controls for immunostaining were carried out by either omitting the primary antibodies, or by incubating with the specific primary antibody pre-absorbed with an excess of the endogenous antigen. Control sections showed no immunostaining in all cases. 


\subsection{Biofunctionalization of gold nanoparticles with $C D 133$ antibody}

GNRs coated with protein $\mathrm{G}$ were used in this study. Currently, the GNR is being extensively used in biomedical applications [13, 32, 33]. The Nanorod type, versus sphere, has been the gold nanoparticle of choice as its synthesis is relatively simple and reproducible, and are routinely used in biomedicine for cancer imaging and therapy. GNRs $(10 \times 40 \mathrm{~nm}$ C12-10-808-TPG-50, NANoPARTz, OD $/ \mathrm{ml}:$ 50) were tuned to the laser source, with a surface plasmon resonance peak (longitudinal band) at $808 \mathrm{~nm}$. Protein $\mathrm{G}$ captures a wide range of Ig's, including rabbit IgG, via the IgG Fc-region. Thus, GNRs were coupled to protein $G$ to facilitate the biofunctionalization with CD133 antibodies. Commercial GNRs-protein G were resuspended thoroughly in PBS to obtain a homogeneous suspension. For the Ig capture, $100 \mu 1$ of GNRs-protein G solution was transferred to a tube at RT and washed in PBS several times by centrifugation $5 \mathrm{~min}$ at $14.5 \mathrm{rpm}$, at RT. Then, polyclonal antibodies against CD133 (St John's laboratory, STJ 20168) were added at a 1:500 final dilution in a solution made of $100 \mu \mathrm{l}$ of washed nanoparticles-protein $\mathrm{G}$ in $500 \mu \mathrm{l} 3 \% \mathrm{BSA}$. The mixture was incubated for $1 \mathrm{~h}$ with gentle rotative agitation, at $4{ }^{\circ} \mathrm{C}$. The mixture was then washed three times in $600 \mu \mathrm{l} 3 \% \mathrm{BSA}$ by centrifugation $5 \mathrm{~min}$ at $14.5 \times 1000 \mathrm{rpm}$, at RT. After the last centrifugation, B-GNRs (GNRs-protein G-CD133 compound) were resuspended in $100 \mu \mathrm{l}$ PBS.

\subsection{Optical hyperthermia device}

A continuous laser wave (MDL H808, PSU-H-LED power source; Changchung New Industries, Changchun Jilin, China) working at $808 \mathrm{~nm}$, with a maximum output power of $5 \mathrm{~W}$, a beam height from base of $29 \mathrm{~mm}$, a beam diameter with an aperture of $5-8 \mathrm{~mm}^{3}$, and a laser head dimensions of $155 \times 77 \times 60 \mathrm{~mm}^{3}$ was used in this study. The laser was connected to the system via a multimode optical fiber with a core diameter of $600 \mu \mathrm{m}$, a length of $1.5 \mathrm{~m}$, and a power transmission of $90 \%-99 \%$ (600 $\mu \mathrm{m}$ MM fiber; Changchung New Industries). The optical fiber was fixed to the stereotaxic device, suspended vertically through a collimation lens (78382, Newport, Irvine, CA) with the downward facing at power density of 3.55 watts $\mathrm{cm}^{-2}$, using a Newport power meter model 843-R with a Newport 818-SL photodetector.

\subsection{Photothermal therapy}

Two weeks after of tumor cell implantation, mice were weighed and then anesthetized with $1 \%$ isofluorane. Randomly selected animals were processed for photothermal therapy. Using the same surgery protocol as in section 2.2 (Mouse tumor model), $2 \mu \mathrm{l}$ of B-GNRs were injected at $4 \mathrm{~mm}$ depth, the same place where the tumor cells were previously injected two weeks before, at a rate of $0.5 \mu 1 \mathrm{~min}^{-1}$. After $5 \mathrm{~min}$, a second dosage of $2 \mu \mathrm{l}$ was then injected at $2 \mathrm{~mm}$ depth by raising the needle appropriately. $10 \mathrm{~min}$ following the second injection, the needle was withdrawn. In summary, a total of $4 \mu \mathrm{l}$ of B-GNRs were injected. The laser was focused through the same burr hole previously used to inject the tumor cells and the B-GNRs. The radiation process was carried out for 25 continuous minutes. The procedure was replicated for three consecutive days to eighteen mice called a treated group, six mice per group (figure 1).

\subsection{Temperature measurements}

A thermal probe (Luxtron Corporation) was introduced into the brain tissue through an additional $1 \mathrm{~mm}$ burr hole (figure 2) in order to analyze the magnitude of the temperature increase following laser irradiation of the B-GNRs particles detected as described in section 4.7. The experiment was carried out in three animals subjected to the same nanoparticle injection protocol and illuminated with a laser for $25 \mathrm{~min}$ Samples were recorded every second using the software supplied by the manufacturer in software LabView 2011 (figure 9).

\subsection{MRI acquisition}

MRI was performed at the Nuclear Magnetic Resonance (NMR) and Electronic Spin Center of the Universidad Complutence de Madrid (UCM) using 1 Tesla benchtop MRI scanner [Icon (1T-MRI); Bruker BioSpin GmbH, Ettlingen, Germany]. The system consists of a $1 \mathrm{~T}$ permanent magnet (without extra cooling required for the magnet) with a gradient coil that provides a gradient strength of $450 \mathrm{mT} \mathrm{m}^{-1}$. The animal monitoring systems and the solenoid mouse head RF coil for the 1T-MRI were integrated into the bed and they allowed the animals to be handled on with accurate positioning of the coil and full control of anesthesia and body temperature. Fifteen minutes before placing the mouse into the MRI bed, mice were intraperitoneally administered with $0.4 \mathrm{mmol} \mathrm{Kg}^{-1}$ Gadopentetate dimeglumine (Gadolinium, Magnevist, Schering, Germany) and then anaesthetized with a mix of isofluorane in oxygen ( $2 \%$ for induction and $1 \%$ for maintenance). In order to evaluate the tumor volume, MRI experiment consisted of two dimensional pre- and post-contrast Fast Spin Echo, T1 and T2 weighted images (T1WI, T2WI). Both sequences were acquired with the same geometry and resolution: field of view of $20 \times 20 \mathrm{~mm}$, number of slices of 15 , slice thickness of $1 \mathrm{~mm}$, matrix size was $160 \times 160$ that was reconstructed to $200 \times 200$, final resolution of $0.100 \times 0.100 \mathrm{~mm}$. T2WI parameters were as follows: repetition time/echo time (TR/TE), 3040/96 ms; 12 averages, echo train length, 12; acquisition time $\sim 8 \mathrm{~min}$. T1WI parameters were: TR/TE, 262/6 ms; 36 averages, echo train length, 2; acquisition time $\sim 12 \mathrm{~min}$. Measurements were performed three and four weeks post CT-2A inoculation. The tumor volume was analyzed using the free software ImageJ 1.50i. In all cases, both absolute and relative tumor volumes, normalized with respect to the total brain volume were measured. The images were repeated one week later for all individuals. In cases showing a clear survival trend, two weekly additional MRI studies were carried out, up to a total of four scans (see figure 1). 


\subsection{Ultrastructural studies}

Experimental tumors treated in vivo by intratumoral injection with gold nanoparticles activated with CD133 (B-GNRs), were processed following a correlated light and electron microscopic procedure [17] to examine the subcellular location of administered gold nanoparticles. For this, animals were fixed as for light microscopy except that the fixative was a mix of $4 \% \mathrm{PF}$ and $0,1 \%$ glutaraldehyde in PBS. The brain was then dissected out, post-fixed in $4 \%$ PF for $4 \mathrm{~h}$., and then washed in $\mathrm{PB} 0.1 \mathrm{M}$. After fixation, tissue frontal sections $40 \mu \mathrm{m}$ thick were obtained using a vibratome (Leica VT1000 $\mathrm{S}$ vibrating blade microtome) and processed for the ultrastructural analysis [17]. Ultrathin sections were obtained with the aid of a Reichert OM U3 ultramicrotome (Reichert, Germany), mounted on formwar-coated grids, stained with lead citrate, and examined under a Jeol 1200 electron microscope (Jeol, Tokyo, Japan) at $80 \mathrm{Kv}$.

\subsection{Statistical analysis}

Statistical Package for the Social Sciences software was used for all statistical tests. When applicable, results are displayed as the mean \pm standard deviation of 3 experiments independent performed. Statistical significance was calculated using the Student's t-test with $p \leqslant 0.05$.

\section{Acknowledgments}

We thank Ms Soledad Martinez (cell culture experiments), Centre for Biomedical Technology (CTB) for technical help. Also, $\mathrm{PhD}$ Teresa López de Mora for critical reading of the manuscript. The authors are thankful to their grant supporters, Universidad Nacional Experimental del Táchira (UNET) Venezuela to Oscar Casanova-Carvajal. This study was also financially supported in part by CIBER-BBN (Spain) and NEUROCENTRO-CM (B2017/BMD-3760) consortium. Characterization of the MNPs has been performed by the ICTS 'NANBIOSIS', Unit 15 (Functional Characterization of Magnetic Nanoparticles) of the CIBER in Bioengineering, Biomaterials \& Nanomedicine (CIBER-BBN) at the Center for Biomedical Technology (CTB) of the 'Universidad Politécnica de Madrid' (UPM). This work was carried out as part of Project PGC2018-(097531-B-I00, funded by the Ministry of Science of Spain.

\section{References}

[1] Stewart B W K P and Wild C P 2014 World Cancer Report 2014 Irvine, California

[2] Gladson C L, Prayson R A and Liu W M 2010 The pathobiology of glioma tumors Annu. Rev. Pathol. Mech. Dis. $533-50$

[3] Wen P Y and Kesari S 2008 Malignant gliomas in adults New Engl. J. Med. 359 492-507

[4] Method R 1969 Grading of astrocytomas Cancer 62 2152-65

[5] Mair D B, Ames H M and Li R 2018 Mechanisms of invasion and motility of high-grade gliomas in the brain Mol. Biol. Cell $292509-15$

[6] Kim E H, Song H S, Yoo S H and Yoon M 2016 Tumor treating fields inhibit glioblastoma cell migration, invasion and angiogenesis Oncotarget 765125

[7] Demuth T and Berens M E 2004 Molecular mechanisms of glioma cell migration and invasion J. Neurooncol. 70 217-28

[8] Kalantari M, Salouti M, Parivar K, Hamidi M and Emadi S 2018 The use of Gold nanorods conjugated with herceptin in breast cancer treatment by photothermal therapy method in mouse model Int. J. Nano Dimens. 9 123-33

[9] Dickerson E B, Dreaden E C, Huang X, El-Sayed I H, Chu H, Pushpanketh S, McDonald J F and El-Sayed M A 2008 Gold nanorod assisted near-infrared plasmonic photothermal therapy (PPTT) of squamous cell carcinoma in mice Cancer Lett. 269 57-66

[10] Heidari Z, Salouti M and Sariri R 2015 Breast cancer photothermal therapy based on gold nanorods targeted by covalently-coupled bombesin peptide Nanotechnology 26 195101

[11] Fernandez Cabada T, Sanchez Lopez de Pablo C, Martinez Serrano A, del Pozo Guerrero F,

Serrano Olmedo J J and Ramos Gomez M 2012 Induction of cell death in a glioblastoma line by hyperthermic therapy based on gold nanorods Int. J. Nanomed. 7 1511-23

[12] Makkouk A R and Madsen S J 2013 Nanoparticle-mediated photothermal therapy of brain tumors Optical Methods and Instrumentation in Brain Imaging and Therapy ed S J Madsen (New York: Springer) pp 235-51

[13] Fernández-Cabada T, Pablo C S-L, de, Pisarchyk L, Serrano-Olmedo J J and Ramos-Gómez M 2016 Optical Hyperthermia using anti-epidermal growth factor receptorconjugated gold nanorods to induce cell death in glioblastoma cell lines J. Nanosci. Nanotechnol. 16 7689-95

[14] Yao C, Zhang L, Wang J, He Y, Xin J, Wang S, Xu H and Zhang Z 2016 Gold nanoparticle mediated phototherapy for cancer J. Nanomater. 2016 1-29

[15] Pourgholi F, Hajivalili M, Farhad J, Kafil H S and Yousefi M 2016 Nanoparticles: Novel vehicles in treatment of glioblastoma Biomed. Pharmacother. 77 98-107

[16] Day E S, Thompson P A, Zhang L, Lewinski N A, Ahmed N, Drezek R A, Blaney S M and West J L 2011 Nanoshellmediated photothermal therapy improves survival in a murine glioma model J. Neurooncol. 104 55-63

[17] Martínez-Murillo R and Martínez A 2007 Standardization of an orthotopic mouse brain tumor model following transplantation of CT-2A astrocytoma cells Histol. Histopathol. 22 1309-26

[18] Binello E, Qadeer Z A, Kothari H P, Emdad L and Germano I M 2012 Stemness of the CT-2A immunocompetent mouse brain tumor model: characterization in vitro J. Cancer 3 166-74

[19] Jiang J X, Chen H W, Yu C, Zhang Y Y, Chen M Y, Tian S and Sun C Y 2015 The promotion of salinomycin delivery to hepatocellular carcinoma cells through EGFR 
and CD133 aptamers conjugation by PLGA nanoparticles Nanomedicine 10 1863-79

[20] Piktel E, Niemirowicz K, Watek M, Wollny T, Deptuła P and Bucki R 2016 Recent insights in nanotechnology-based drugs and formulations designed for effective anti-cancer therapy J. Nanobiotechnol. 14 1-23

[21] Abadeer N S and Murphy C J 2016 Recent progress in cancer thermal therapy using gold nanoparticles J. Phys. Chem. C 120 4691-716

[22] Jain P K, Huang X, El-Sayed I H and El-Sayed M A 2008 Noble metals on the nanoscale: optical and photothermal properties and some applications in imaging, sensing, biology, and medicine Acc. Chem. Res. 41 1578-86

[23] Zimmerman H M and Arnold H 1943 Experimental brain tumors: II. Tumors produced with benzpyrene Am. J. Pathol. $19939-55$

[24] Sugawara K I, Kurihara H, Negishi M, Saito N, Nakazato Y, Sasaki T and Takeuchi T 2002 Nestin as a marker for proliferative endothelium in gliomas Lab. Invest. 82 345-51

[25] Le Fèvre R et al 2017 Enhanced antitumor efficacy of biocompatible magnetosomes for the magnetic hyperthermia treatment of glioblastoma Theranostics 7 4618-31

[26] Thrall D E, Rosner G L, Azuma C, Larue S M, Case B C, Samulski T and Dewhirst M W 2000 Using units of CEM $43{ }^{\circ} \mathrm{C}$ T 90 , local hyperthermia thermal dose can be delivered as prescribed Int. J. Hyperth. 16 415-28

[27] Abate L E, Mukherjee P and Seyfried T N 2006 Gene-linked shift in ganglioside distribution influences growth and vascularity in a mouse astrocytoma $J$. Neurochem. 98 1973-84

[28] Lu G, Wai S M, Poon W S and Yew D T 2005 Ki67 and doublecortin positive cells in the human prefrontal cortices of normal aging and vascular dementia Microsc. Res. Tech. $68255-7$

[29] Hsu S M, Raine L and Fanger H 1981 The use of antiavidin antibody and avidin-biotin-peroxidase complex in immunoperoxidase technics Am. J. Clin. Pathol. 75 816-21

[30] Rodrigo J, Springall D R, Uttenthal O, Bentura M L, Abadia-Molina F, Riveros-Moreno V,

Martínez-Murillo R and Polak S M J M 1994 Localization of nitric oxide synthase in the adult rat brain Phil. Trans. $R$. Soc. B 345 175-221

[31] Muñetón-Gómez V C, Doncel-Pérez E, Fernandez A P, Serrano J, Pozo-Rodrigálvarez A, Vellosillo-Huerta L, Taylor J S, Cardona-Gómez G P, Nieto-Sampedro M and Martínez-Murillo R 2012 Neural differentiation of transplanted neural stem cells in a rat model of striatal lacunar infarction: light and electron microscopic observations Front. Cell. Neurosci. 6 1-19

[32] Kim D-H, Kim K-N, Kim K-M and Lee Y-K 2009 Targeting to carcinoma cells with chitosan- and starch-coated magnetic nanoparticles for magnetic hyperthermia J. Biomed. Mater. Res. A 88A 1-11

[33] Avvakumova S, Colombo M, Tortora P and Prosperi D 2014 Biotechnological approaches toward nanoparticle biofunctionalization Trends Biotechnol. 32 11-20 